\title{
Feminización de la judicatura española
}

The feminization of the Spanish judiciary

\author{
Carlos Gómez-Bahillo / cgomez@unizar.es \\ Carmen Elboj-Saso / celboj@unizar.es \\ Universidad de Zaragoza, España \\ Celia Marcén-Muñío / celia.marcen@gmail.com \\ Universidad San Jorge, Zaragoza, España
}

\begin{abstract}
The Spanish judiciary is undergoing a major transformation, in which women have been gaining prominence, being majority in the latest promotions. However, women have been discriminated in their access to high responsibility positions, in which their representation is low, even though they meet all the requirements. Presumably, in the coming years, the presence of women indecision-making bodies and the judicature's management will substantially increase. As a result, justice could become more effective and efficient The methodology used was, on the one hand, descriptive and analytic, considering the increasing participation of women in the Spanish judiciary and analyzing the feminization of the judicature professionals; and, on the other, qualitative, collecting their views on this phenomenon and on the existing conditions of equality in the Spanish judicial system.
\end{abstract}

Key words: feminization, glass ceiling, opportunities, discrimination.

Resumen: La judicatura española está experimentando una transformación importante, y en las últimas promociones las mujeres superan a los hombres. No obstante, hasta fechas muy recientes la mujer ha estado discriminada en el acceso a puestos de máxima responsabilidad, donde su representación es reducida, a pesar de reunir las condiciones requeridas. Previsiblemente, en los próximos años la presencia de la mujer en los órganos de decisión y gestión judicial se habrá incrementado sustancialmente y, como consecuencia de ello, tal vez la justicia sea más eficaz y eficiente. La metodología utilizada es de carácter cuantitativo de tipo descriptivo y analítico, dado que se estudia el aumento creciente de la presencia de mujeres dentro de la carrera judicial española, y se hace un análisis del proceso de feminización de los profesionales de la justicia. Y también de carácter cualitativo, recogiendo sus opiniones sobre este fenómeno y las condiciones de igualdad existentes en la organización judicial española. Palabras clave: feminización, techo de cristal, oportunidades, discriminación. 


\section{Introducción}

La presente investigación se sitúa en el campo de la sociología de las organizaciones públicas y tiene como objetivo demostrar el proceso de feminización que está experimentando la administración judicial española, y cómo las mujeres que están accediendo a la carrera judicial están ocupando puestos de primer nivel -jueces de primera instancia e instrucción, juzgados de los social-, siendo su presencia todavía reducida en los de máxima responsabilidad y en los órganos superiores de la magistratura. Para ello, se da respuesta a las siguientes cuestiones:

¿La presencia de mujeres que se está produciendo en estos últimos años, y que va en aumento, significa que existe una igualdad de oportunidades en la institución judicial española, o ésta únicamente se da en el acceso, reservándose los puestos de mayor relevancia para los varones? ¿La posibilidad de llegar a los puestos de responsabilidad por parte de las mujeres está condicionada por la dificultad de conciliar vida profesional y familiar, o continúa existiendo por parte de los órganos institucionales una preferencia favorable a la designación de varones?

El interés de la investigación reside en que la carrera judicial española ha pasado de ser una organización reservada exclusivamente para los varones a ser en la actualidad superior el número de mujeres que forman parte de ella. ¿Se trata de un cambio solamente cuantitativo o es más profundo y está afectando a las estructuras del poder judicial y se está produciendo una democratización dentro de él?

\section{Planteamiento metodológico}

El objetivo general de este estudio es presentar el proceso de feminización de la judicatura española, a través del desarrollo de tres hipótesis de partida, que constituyen el hilo conductor de la investigación.

$1^{\text {a }}$ La carrera judicial es una institución donde no existe discriminación en los momentos de acceso, y las mujeres universitarias tienen mayores posibilidades de desarrollar un trabajo cualificado, afín a su especialización.

$2^{\text {a }}$ Para acceder a los puestos más relevantes de la cúpula judicial se requiere una mayor disponibilidad, por lo que la mujer con obligaciones familiares, en la práctica, tiene menos posibilidades que sus compañeros varones para acceder a puestos de mayor responsabilidad. 
$3^{\text {a }}$ En estos últimos años se está produciendo un cambio importante en la judicatura española con la aprobación de normas tendentes a equiparar las oportunidades de las jueces y magistradas con las de sus compañeros, y que como resultado ha permitido la presencia paulatina de mujeres en órganos de responsabilidad y decisión.

La metodología utilizada en esta investigación es de carácter descriptivo y analítico, ocupando las técnicas del análisis documental y la opinión de los propios interesados.

Los documentos considerados para el análisis cualitativo han sido: $1^{\circ}$ ) VI Barómetro de Opinión de la Judicatura Española que recoge 401 entrevistas distribuidas de forma proporcional al número de jueces y magistrados de toda España, con selección aleatoria de juzgados según orden de jurisdicción y dentro de ellos selección aleatoria de jueces y magistrados española (excluyendo al Tribunal Supremo), siendo la muestra representativa de la carrera judicial, por razón de género. $2^{\circ}$ ) V Encuesta a la Carrera Judicial. $3^{\circ}$ ) Encuesta a la carrera judicial sobre conciliación de la vida familiar, en la cual han participado 751 miembros de la carrera judicial. $4^{\circ}$ ) Asimismo, se recoge la opinión de mujeres que están ocupando puestos de responsabilidad y describen las dificultades que han tenido, tanto en el ámbito institucional como personal y familiar.

Para el análisis cuantitativo se ha utilizado la información estadística oficial proporcionada por el Consejo General del Poder Judicial mediante su publicación anual Informe sobre la estructura demográfica de la Carrera Judicial.

\section{Contextualización. La mujer cualificada y su inserción profesional}

Las sociedades y grupos humanos a lo largo de su historia han generado relaciones asimétricas de poder entre hombres y mujeres, a partir del establecimiento de roles diferenciadores en función del género, lo cual ha contribuido a privar a la mujer de las mismas oportunidades que tiene el varón, para ocupar puestos de importancia en organizaciones e instituciones, al prevalecer unos valores que minoran su capacidad para el desempeño de determinadas funciones de responsabilidad a causa de sus obligaciones domésticas.

Esta división sexualizada de roles entre géneros, y los principios en los cuales se fundamenta, ha sido estudiada por Bourdieu (2005) como una construcción social basada en las diferencias biológicas entre hombre y mujer, lo que ha justificado histórica y socialmente la dominación masculina, y 
que han determinado las relaciones sociales entre ambos géneros. Pero esta dominación, que ha denominado violencia simbólica, se traduce en la imposición del hombre sobre la mujer en todos los ámbitos relacionales (doméstico, profesional, social, etc.), y en la asunción y asimilación por parte de ellas de esta situación social, que les lleva finalmente a aceptar un orden social sexuado, el cual empieza dentro de la propia familia, pues se educa en ello, y que trasciende posteriormente al mundo laboral.

El "poder" es un concepto inherente a las relaciones de género por las siguientes razones:

A) En primer lugar, el género y el poder están vinculados estructuralmente: la representación de los hombres, en general, es más alta en las ocupaciones con salarios más altos, y con más poder formal, organizativo, político e institucional. B) En segundo lugar, género y poder están vinculados culturalmente, en las prácticas sociales, tareas, posiciones y características que se construyen socialmente según el género (...) Estos vínculos resaltan la distribución de desigual de poder entre mujeres y hombres y han sido parte integral de la reformulación feminista de género en términos del construccionismo social... (Brunet et al., 2011: 41).

Esta discriminación/segregación laboral de la mujer se produce principalmente a lo largo de tres momentos de su vida:

- Elección de carrera. Los estudios universitarios o profesionales que mayor relevancia social y, generalmente también, con superiores retribuciones económicas tienen una mayor presencia masculina, mientras que en las carreras de carácter social y cuya actividad requiere más habilidades y competencias sociales predominan mujeres (Navarro-Guzmán y Casero-Martínez, 2012).

- Acceso al mercado laboral. A igualdad de condiciones académicas y capacitaciones profesionales, la mujer tiene dificultades para poder competir con el varón, a quien por el hecho de serlo se pone por delante de ella.

- Desarrollo profesional. Se sigue manteniendo la idea de que los puestos en los que se deben tomar decisiones, y especialmente aquellas con implicaciones o efectos sobre otras personas o sobre sus bienes, deben ser ocupados por hombres, dado que ellos son más objetivos y se dejan llevar menos por sentimientos (Sarrio et al., 2002). Además, si la mujer es joven y puede quedarse embarazada encuentra múltiples dificultades para asumir puestos de dirección y gestión.

Estos estereotipos en torno a las masculinidad/feminidad han sido incorporados a la cultura popular y se mantienen a lo largo del tiempo, a pesar de los signos de modernidad introducidos en las sociedades occidentales, 
determinando identidades y costumbres sociales, estilos de vida, actitudes, etc. Culturalmente se ha otorgado a la mujer los roles internos de cuidado, mientras que al hombre se le han reservado los roles de representación familiar y social.

El directivo ideal responde a un prototipo de persona: disponible, entregado totalmente a su tarea, sin preocupaciones familiares, etc., situación que socialmente se asocia casi siempre con la figura del varón. Por tanto, la tipología ideal (Weber, 1993) existente en el imaginario colectivo discrimina a la mujer para acceder a puestos de responsabilidad y decisión, no por falta de cualidades o formación, sino por tener menor disponibilidad.

Romper con estos estereotipos ha sido un largo proceso, y gracias a las luchas, principalmente del movimiento feminista, poco a poco se van recortando las diferencias sociales que situaban a las mujeres en un segundo plano y superando las múltiples barreras que dificultaban su plena participación en ámbitos como el educativo o el laboral (Beck-Gernsheim et al., 2001).

En la actualidad las mujeres en las sociedades desarrolladas aspiran a tener las mismas oportunidades educativas, formativas y laborales que los hombres.

En primer lugar, las mujeres se ubican mayoritariamente en las ramas de producción de servicios, principal fuente de creación de empleo en nuestro mercado de trabajo. En segundo lugar, se observa una clara coincidencia entre la evolución de la estructura de ocupaciones y la distribución femenina. En tercer lugar, las mujeres intensifican sus inversiones en educación, en consonancia con las mayores exigencias de cualificación de las actividades productivas (Castaño et al., 1999: 17).

No obstante, las mujeres que han alcanzado un nivel de cualificación superior a la media masculina continúan siendo relegadas a ocupar puestos de menor responsabilidad y decisión. Existe un "techo de cristal"1 que impide a las mujeres ocupar puestos de responsabilidad a pesar de tener los conocimientos y competencias profesionales requeridas para ello. SánchezApellániz (1997) distingue tres barreras inherentes a este "techo de cristal" que sufre la mujer:

1 El término "techo de cristal", o "Glassceilingbarriers" en el original en inglés, apareció por primera vez en un artículo del Wall Street Journal en 1986 en Estados Unidos, y se utilizó para designar a las barreras invisibles con las que se encuentran las mujeres cualificadas en el desarrollo de su carrera profesional. A partir de entonces ha sido usado con frecuencia en estudios de sociología de trabajo y sociología de la mujer para analizar los impedimentos con los que se encuentran las mujeres para ocupar puestos de responsabilidad en las instituciones públicas y privadas. Comenzaron a hacer uso de este concepto, entre otros, Carr-Ruffino (1991), Lynn (1991), Davison y Cooper (1992), Holloway (1993), etcétera. 
- Las barreras internas relacionadas con la identidad social del género. Desde el inicio de la vida se produce un proceso de socialización -a través de la familia, escuela con el currículum oculto, medios de comunicación, etc. - diferenciado y vinculado al género, y se educa a los niños/jóvenes en una sociedad con roles atribuidos en función del mismo, que responden a actitudes y expectativas establecidas socialmente y discriminatorias para las mujeres.

- Las barreras externas vinculadas a la cultura organizacional y a la existencia de estereotipos de género. Y como consecuencia de ello, la distinción de roles y desempeño de funciones y actividades diferenciadas por razón de género se encuentran vigentes en la cultura de las organizaciones, al estar dominadas por valores androcéntricos que actúan siempre en contra de la promoción de la mujer.

- Barreras derivadas de su rol familiar (procreación y responsabilidades domésticas). La necesidad de conciliar la actividad profesional con la responsabilidad doméstica constituye un impedimento para que la mujer pueda desempeñar puestos con horarios más inestables o que requieren movilidad. La falta de un reparto equitativo de las tareas domésticas en el ámbito de la pareja contribuye indirectamente a ello.

La mujer se encuentra habitualmente con dificultades para poder conciliar vida familiar y profesional por la falta de medidas públicas, por el peso de la cultura masculina en la mayor parte de las organizaciones, tanto públicas como privadas, y por la carencia de sensibilidad social para ver la desigualdad como una mera situación de hecho pero no como problema. Es necesario transformar esta situación y promover un nuevo orden relacional entre géneros, principalmente en el mercado laboral (Wirth, 2002).

La segregación horizontal ha permitido y favorecido la concentración de mujeres en algunos sectores de actividad, ocupando mayoritariamente puestos de responsabilidad media, y casi siempre relacionados con sus roles tradicionales, en departamentos y organizaciones de administración y servicios dedicados a actividades de carácter social (Sarrio et al., 2002), teniendo que convertirse en trabajadoras "genéricas" (flexible, con capacidad de adaptación a horarios y a distintas tareas, sustituible por otra que acepte las condiciones de sobreexplotación...); es la tendencia mayoritaria de las mujeres que se incorporan al mercado laboral globalizado (Amorós, 2005).

Cobo (2010) señala que la globalización económica está haciendo crecer el empleo y el trabajo de las mujeres; sin embargo, dicha incorporación no marca una tendencia radical de cambio ni tampoco produce los mismos efectos para todas las mujeres. 
La discriminación por razón de género es menor en la administración pública en relación con la empresa privada, dado que la mujer puede acceder a puestos cualificados con mayores niveles de retribución e idénticos a los de sus compañeros varones, mediante pruebas selectivas. No obstante, también en ellas ha existido y existe una discriminación encubierta para el acceso a puestos de responsabilidad y que únicamente una minoría muy cualificada de mujeres consigue ocupar. "Existe un tope invisible que impide a las mujeres llegar en el terreno público, donde están los hombres. Es lo que se ha denominado techo de cristal, que oculta una discriminación indirecta, no reflejada en las leyes y que se mide por los resultados diferenciales" (Osborne, 2005: 163).

Conseguir la igualdad real de oportunidades es un largo proceso que comienza por la eliminación de las discriminaciones existentes en al ámbito legislativo y en el acceso a la educación superior, y promover procedimientos que tiendan a la igualdad social y material respecto a los hombres (Barberá, 2005). Y a ello habrá que añadir en el ámbito doméstico, con una distribución más igualitaria de roles y funciones entre la pareja como señala Miller (2004: 59), "la incorporación de la mujer al mercado de trabajo no depende de una intervención voluntaria por parte del Estado o de una presión a través de las reivindicaciones, sino de una transformación en el ámbito doméstico que afecta, entre otros aspectos, al estatus sociolaboral que tradicionalmente han desempeñado los hombres".

\section{Mujer en la administración de justicia}

La administración de justicia está experimentando una importante transformación como consecuencia del acceso de la mujer a puestos de jueces, magistrados, fiscales y administradores judiciales, si bien los niveles de responsabilidad de la cúpula judicial continúan siendo ocupados todavía, en la mayoría de las ocasiones, por hombres. Este proceso de feminización se está produciendo durante las últimas décadas en muchos países europeos y latinoamericanos, y ha dado lugar a una amplia bibliografía, en la que se ha estudiado las razones y causas de este cambio en el poder judicial, una institución que durante muchos siglos ha estado reservada a los hombres.

Entre estos estudios destacamos: Perry (1991) considera la representación femenina en los órganos judiciales de Estados Unidos; Flores (2012) analiza desde una perspectiva sociológica lo que está suponiendo la presencia de la mujer en la administración de justicia de México; Bustillos (2011) estudia la estructura y funcionamiento de la carrera judicial en México; Cavagna 
et al. (1994) y Gastiazoro (2008) destacan el aumento de la presencia femenina y el proceso de transformación que ello está suponiendo en la administración de la justicia argentina; Kohen (2008) analiza la presencia femenina y sus efectos en la administración justicia argentina; Rueda (2009) estudia el incremento de juezas en la administración de justicia en Perú, partiendo de la teoría de género; Boigeol (2007) considera las dificultades con las se encuentran juezas y magistradas para acceder a puestos de responsabilidad en Francia, etcétera.

A la administración de justicia acceden mujeres con elevados niveles de formación, tras superar en la mayoría de las ocasiones unas pruebas de acceso difíciles y complejas, que requieren un gran esfuerzo personal, capacidad, constancia y fuerza de voluntad. Por eso, es una institución atractiva para las mujeres competentes dado que en ella pueden desarrollar su capacidad y demostrar su valía y, por tanto, sentirse reconocidas y gratificadas por el esfuerzo y trabajo realizado, lo que no ocurre en la mayoría de las ocasiones en las empresas privadas (Junqueira, 2003).

Suele ser una ocupación en la que se encuentran satisfechas y generalmente no se sienten discriminadas, si bien admiten sus dificultades tanto institucionales como personales -compaginar su trabajo con sus obligaciones familiares- para acceder a los puestos de mayor responsabilidad. Algunos autores, como Kohen (2008) en Argentina y Junqueira (2003) en Brasil, destacan además que este aumento de mujeres en la institución judicial se debe a la mayor facilidad para compaginar las obligaciones y tareas domésticas con el ejercicio profesional, dada su discrecionalidad para realizar y organizar su trabajo.

No obstante, no es tan igualitaria respecto al varón la posición de la mujer en los procesos de acceso y selección, como destaca Bergallo (2006), refiriéndose a los procesos de reclutamiento en Argentina, especialmente para los puestos de máxima responsabilidad. Por ello, las mujeres deben establecer estrategias para cambiar la inercia de la discriminación y dominación por parte del varón en la administración de justicia, como señala Boigeol (2007), al referirse al sistema judicial francés. Sobre la diferente aportación de la mujer al proceso judicial, por su sensibilidad y escala de valores, hay que destacar los estudios realizados por Malleson (2007) en el sistema judicial italiano, Menkel-Meadow (2002) en Estados Unidos, Gastrón (2009) en Argentina, Fix-Fierro y López-Ayllon (2006) en México, etcétera. 


\section{Mujer en la judicatura española}

Hasta hace cuatro décadas la carrera judicial en España ${ }^{2}$ ha sido una institución de hombres, al igual que sucedía en la mayoría de los países. Durante la etapa franquista, el Fuero de los Españoles reconocía la no discriminación por razón de sexo y estado en el desarrollo de los derechos laborales y profesionales (artículo 11: “...todos los españoles podrán desempeñar cargos y funciones públicas según su mérito y capacidad"). No obstante, la mujer era discriminada de la carrera judicial, dado que la Ley del 18 de diciembre de 1950 por la que se regula la Escuela Judicial — creada por Ley del 26 de mayo de 1944 - establecía que para poder acceder a la misma era condición ser varón.

La Ley 56/1961, del 22 de julio, sobre derechos politicos profesionales y de trabajo de la mujer suprime las restricciones y discriminaciones existentes y reconoce el derecho de la mujer a acceder a los distintos cuerpos de la administración pública, reconociendo su capacidad para desarrollar cualquier actividad, excepto aquellas que requieren una fuerza desmesurada.

Esta ley supuso un avance, ya que prohibió toda forma de discriminación laboral en función del sexo, y expresamente la salarial, y a partir de la misma las reglamentaciones laborales suprimieron las cláusulas de despido por razón de matrimonio, vigente hasta entonces. No obstante, se mantuvo la discriminación al impedir el acceso de la mujer a determinadas funciones de la administración del estado, como se recoge en su artículo tercero dos: "Se exceptúan de los dispuesto (...) el ingreso en: (...) c) La Administración

2 La carrera judicial en España está formada por jueces, magistrados y fiscales. Los jueces y fiscales son seleccionados a través de una oposición libre en la que pueden participar los mayores de edad, sin antecedentes penales, que sean titulados en derecho y gocen de la plenitud de sus derechos civiles. En el caso de los candidatos a la judicatura, una vez aprobadas las pruebas deben superar un curso de capacitación en la Escuela Judicial, cuya duración es de dos años y, una vez conseguido, son nombrados jueces. Los fiscales realizan también un curso formativo en el Centro de Estudios Jurídicos. Los magistrados son los jueces que sirven en órganos judiciales superiores, como las Audiencias Provinciales, los Tribunales Superiores de Justicia o la Audiencia Nacional. Las plazas de magistrado se cubren con arreglo a un sistema de cuotas entre los profesionales de la justicia y el derecho. Los magistrados son adscritos a los diferentes tribunales con arreglo a normas específicas que tiene en cuenta criterios técnicos y de especialización. La promoción a la categoría de magistrado del Tribunal Supremo se lleva a cabo con arreglo a criterios similares.

Dentro de cada categoría, los presidentes de los respectivos órganos jurisdiccionales y de las salas en que se divida cada uno son nombrados por el Rey mediante Real Decreto, a propuesta del Consejo General del Poder Judicial. 
de Justicia en los cargos de Magistrados, Jueves y Fiscales, salvo en las jurisdicciones tutelar de menores y laboral". Hay que esperar a las reformas de los Códigos Civil y de Comercio, en 1975 3/4año en el que desaparece la dictadura franquista $3 / 4$ para que la autorización marital para firmar un contrato laboral y ejercer el comercio fuese abolida.

La Ley de 1961 fue elaborada por una élite tecnocrática gubernamental que pretendía mejorar la imagen de España en el contexto internacional, y a pesar del régimen dictatorial, mostrar la situación de la mujer española, respecto a sus posibilidades laborales, equiparable a la de las sociedades europeas, y para ello se derogaron los impedimentos legales que les imposibilitaba acceder a determinados puestos en la administración pública (Telo, 1966: 81).

A lo largo de 1966 se planteó la necesidad de derogar los artículos de la Ley de 1961 que excluía a la mujer de la carrera judicial, por la presión social de colectivos de mujeres universitarias vinculadas a las Facultades de Derecho, lo cual tuvo un importante impacto mediático en los medios de comunicación (Espuny et al., 2010: 111). Las cifras de alumnas matriculadas en las Facultades de Derecho en España son un claro indicio de ello, y su aumento ha sido progresivo desde entonces: 1959-1960 (855 alumnas, 5,8\%), 1975-1976 (14.277 alumnas, 30,1\%), 1980-1981 (31.123 alumnas, 37.3\%), 1990-1991 (99.323 alumnas, 53,3\%), 2000-2001 (81.305 alumnas, 52,2\%), 2010-2011 (34.537 alumnas, 50,9\%) (Instituto Nacional de Estadística).

Debe tenerse en cuenta que por aquellos años, y como consecuencia del incremento de la renta personal y familiar y la consolidación de una clase media urbana, comienza a aumentar el número de mujeres que acceden a los estudios universitarios para cursar no solamente carreras de carácter humanístico, generalmente muy feminizadas, sino otras como derecho, economía, empresariales, etc.; principalmente las hijas de profesionales o empresarios.

La feminización de la carrera de derecho supuso un aumento de la presencia de mujeres en empresas y despachos profesionales, aunque en la mayoría de las ocasiones ocupando puestos de inferior cualificación por sus funciones y condiciones económicas que el que le correspondería por su titulación. Desempeñar un puesto en la administración pública permitió a la mujer poder competir con el varón en igualdad de condiciones, abriendo un importante nicho laboral para las mujeres cualificadas, aunque continuó existiendo discriminación para acceder a puestos de máxima responsabilidad, cuya presencia femenina es muy escasa en aquellos años (y cuando lo consigue es a organismos de carácter social o asistencial, pero no en aquellos en los que se toman decisiones económicas o que están más relacionados con la productividad). 
La Ley 96/1966, del 28 de diciembre, suprime la limitación establecida en el apartado c) del número dos del artículo tercero de la Ley del 22 de julio de 1961 sobre derechos de la mujer, y le permite el acceso a los cuerpos de magistrados, jueces y fiscales de la administración de justicia. Este cambio normativo se justifica porque, según se señala en el Preámbulo de la Ley, "tal excepción respondió, sin duda no a la idea de una falta de capacidad o responsabilidad de la mujer para desempeñar tales cargos, sino más bien a una protección de sus sentimientos ante determinadas actuaciones que el cumplimiento del deber haría ineludibles". Esto se produce en un momento político de aparente apertura política por parte del gobierno de la nación que intenta por todos los medios su integración en Europa.

En la actualidad, en las últimas promociones de acceso a la carrera judicial las mujeres superan a los hombres. El análisis de las cifras de los informes anuales sobre la estructura demográfica de la Carrera Judicial nos indica este proceso de cambio. El acceso de la mujer a la judicatura española se ha producido principalmente durante las tres últimas décadas, ${ }^{3}$ si bien esta tendencia ha ido en aumento, y los ingresos producidos en 2014 lo confirman, dado que el número de mujeres por el turno libre ${ }^{4}$ supuso el 63,2\% del total. En el caso del turno distinto al libre accedieron dos varones por cada mujer.

Si consideramos la forma de acceso de los profesionales que actualmente forman la carrera judicial española, observamos que en el caso de las mujeres se produce mayoritariamente a través de turno libre (96,6\%), generándose una discriminación respecto a las otras formas de ingreso, especialmente como "jurista de reconocida competencia" (15-20 años de ejercicio profesional), a pesar de que existen mujeres juristas en la abogacía española con

3 El 23 de enero de 1978, Josefina Triguero Agudo tomaba posesión de su plaza en el Juzgado de Navalmoral de la Mata, en Badajoz, y se convertía en la primera juez de España. No obstante, la primera juez fue Concepción de Carmen Venero, miembro del Tribunal Tutelar de Menores, quien previa oposición en el Cuerpo de Oficiales del Tribunal Tutelar de Menores ascendió a secretario habilitado y posteriormente fue nombrada por el Ministerio de Justicia juez de dicho tribunal. Recordemos que la Ley de 1961 permitía acceder a las mujeres al puesto de juez exclusivamente en las jurisdicciones tutelar de menores y laboral.

$4 \mathrm{El} \mathrm{Turno} \mathrm{libre} \mathrm{consiste} \mathrm{en} \mathrm{la} \mathrm{superación} \mathrm{de} \mathrm{unas} \mathrm{pruebas,} \mathrm{conocidas} \mathrm{como} \mathrm{oposiciones,} \mathrm{en}$ las que el candidato debe demostrar sus conocimientos jurídicos y su capacidad de aplicación e interpretación de las leyes. En el turno restringido, para el que se reservan un número reducido de plazas, acceden a la judicatura profesionales de reconocido prestigio, con más de diez años de ejercicio profesional, y en este caso se tienen en cuenta únicamente sus méritos personales y trayectoria profesional, sin que tengan que realizar ninguna prueba específica; los seleccionados hacen un curso de formación común a los de turno libre. 
reconocimiento internacional, lo cual demuestra las dificultades con las que se encuentra la mujer para poder competir no por falta de currículum, sino por la conciliación entre obligaciones profesionales y familiares.

En el caso del turno jurídico militar la razón es por la escasa vinculación de la mujer abogada con los temas jurídicos relacionados con las Fuerzas Armadas. Estas diferencias por razón de género continúan manteniéndose, como se puede comprobar en la Tabla $1^{5}$ (periodo 2005-2014), a pesar del aumento del número de mujeres en la carrera judicial.

La edad media de los miembros de dicha carrera confirma nuevamente esta tendencia a la feminización, pues resulta superior el número de mujeres jueces y magistradas menores de 50 años, mientras que entre los mayores de esa edad son principalmente varones (Tabla 2); esto se debe a que el aumento de mujeres que han accedido a la judicatura se ha producido mayoritariamente durante los últimos años. En conjunto, observamos un poder judicial relativamente joven, dado que el $61,7 \%$ de sus miembros no sobrepasa la edad de los 50 años; siendo estas diferencias significativas entre géneros, ya que es más joven el colectivo de mujeres.

La comparación entre los datos correspondientes a 2005 con los de 2014 nos muestra el cambio, y cómo cada vez más la mujer se está posicionando al lograr ocupar puestos de mayor responsabilidad dentro de ella. La carrera judicial tiene reconocido un prestigio y estatus social, y unas retribuciones más elevadas a las medias de los titulados superiores que trabajan para la administración, y unas condiciones laborales más favorables respecto a la distribución del trabajo, horario, etc., sin que existan diferencias en ello por razón de género.

A pesar de ser mayoría, las mujeres todavía son minoritarias en los órganos judiciales superiores, como la Audiencia Nacional, los Tribunales Superiores de Justicia de las Comunidades Autónomas, el Tribunal Supremo o jueces decanos, a pesar de haber transcurrido ya cuarenta años desde que se les permitió el acceso a la administración de justicia. Su presencia es superior en los juzgados, puestos que se ocupan desde el inicio de la carrera judicial (véase Tabla 3), y como señala Robles (2004: 48):

La presencia de la mujer muy equiparada con la de los hombres en los distintos Juzgados que se reparten a lo largo y ancho de la geografía española, por los diferentes partidos judiciales, sin que se aprecie por razón del tipo de Juzgado (Primera Instancia,

5 Todas las tablas y los cuadros se encuentran en el Anexo, al final del presente artículo (Nota del Editor). 
Instrucción, Mixtos, Familia, Penal, Social, Contencioso, Menores, Vigilancia Penitenciaria) ninguna tendencia a que la mujer, por el hecho de ser mujer, pida o tenga preferencia por unos u otros en función de las materias de las que conocen, pues Juzgados como los de Familia o Menores, son ocupados indistintamente por hombres o mujeres, igualmente sucede en las Audiencias Provinciales.

Si consideramos los Órganos Centrales del Poder Judicial (Tabla 3) la presencia de mujeres es reducida, siendo la diferencia respecto a los hombres significativa, a pesar de haber ya un número importante de mujeres que tienen suficientes años de experiencia y currículum profesional para ser designadas para estos puestos de responsabilidad en los tribunales superiores (Audiencia Nacional y presidencia de Tribunales Superiores de las Comunidades Autónomas y Audiencias Provinciales). Como señala Ribas (2014), la presencia de las mujeres en sectores tradicionalmente masculinos, y en especial en las posiciones de poder y responsabilidad históricamente asignadas a los hombres, no se ha incrementado al mismo ritmo, produciéndose una segregación vertical en referencia a la poca representación de las mujeres en los niveles jerárquicos superiores.

No obstante, esta situación ha comenzado a cambiar en los últimos años tras la aprobación de la Ley Orgánica 3/2007, del 22 de marzo, para la igualdad efectiva de mujeres y hombres, en cuyo artículo 16 establece que "los poderes públicos procurarán atender al principio de presencia equilibrada de mujeres y hombres en los nombramientos y designaciones de los cargos de responsabilidad que les correspondan”. El Plan Estratégico de Igualdad de Oportunidades (2008-2011), aprobado en Consejo de Ministros el 14 de diciembre 2007, establece como objetivo estratégico 3: "Incrementar la participación de las mujeres en los puestos de dirección y responsabilidad en el poder judicial", y para ello promueve el desarrollo de las Comisiones previstas en la citada Ley Orgánica 3/2007, del 22 de marzo.

Para favorecer la igualdad de oportunidades en la administración de justicia, el Consejo General del Poder Judicial aprobó un Reglamento, en febrero de 2010, que regula la provisión de plazas de nombramiento discrecional en los órganos judiciales.

Existe una demanda entre jueces y magistrados para que se establezca un sistema más objetivo en la adjudicación de estos puestos de responsabilidad superior, como se refleja en la $V$ Encuesta a la Carrera Judicial ${ }^{6}$ (Cuadro 2),

6 VEncuesta a la Carrera Judicial. Elaborada a propuesta del Consejo General del Poder Judicial, se realizó en 2010, a través de un cuestionario autoadministrado, remitido por correo, a 4.528 jueces y magistrados. Los cuestionarios respondidos fueron 1.763 , lo que supone un 
donde se propone una modificación de los criterios para la designación por parte del Consejo General del Poder Judicial de los magistrados del Tribunal Supremo y la presidencia de sus Salas; la presidencia de la Audiencia Nacional y de sus Salas; los magistrados y presidencia de los Tribunales Superiores de Justicia de las Comunidades Autónomas y de sus Salas, y las presidencias de las Audiencias Provinciales. Y se prime preferentemente la experiencia en el orden jurisdiccional y en el órgano colegiado, así como la antigüedad en el escalafón y en el órgano colegiado que se pretende presidir, y se dé menos valoración a la entrevista del candidato para la exposición de sus méritos e idoneidad.

La Resolución del 20 de mayo de 2011, de la Secretaria de Estado para la Función Pública, por la que se publica el Acuerdo del Consejo de Ministros del 28 de enero de 2011, por el que se aprueba el I Plan de Igualdad entre mujeres y hombres en la Administración General del Estado y en sus Organismos Públicos, establece dentro del Eje 2 como uno de los objetivos más importantes del Plan: "Promover y mejorar las posibilidades de acceso de las mujeres a puestos de responsabilidad".

Para poner fin a esta situación y cumplir con lo establecido por las disposiciones aprobadas para favorecer la igualdad de oportunidades por razón de género, el propio Consejo General del Poder Judicial aprobó, el 14 de febrero de 2013, un Plan de Igualdad de la Carrera Judicial para conseguir la igualdad efectiva entre hombres y mujeres en los órganos judiciales, y concretamente facilitar la promoción de las mujeres con méritos y capacidad para cargos gubernativos de la carrera, así como magistradas del Tribunal Supremo.

Además el plan establece once ejes estratégicos de actuación del Consejo General del Poder Judicial para favorecer la igualdad en numerosos aspectos, siendo los más relevantes: el acceso a la carrera judicial por el turno restringido, la promoción profesional y formación profesional, la sensibilización y conciliación con la vida familiar y la mayor participación en los órganos de decisión. Se están tomando medidas, como podemos observar en las memorias e informes anuales; si bien sus efectos van a un ritmo más lento que el deseado.

En la actualidad, el Consejo General del Poder Judicial está compuesto por 21 magistrados, de ellos nueve son mujeres, lo cual supone un cambio importante teniendo en cuenta que durante los años 2001-2008 únicamente

$39 \%$, y a pesar de no haberse utilizado un muestreo probabilístico, los participantes reflejan el universo de estudio considerado en cuanto a rasgos sociodemográficos. 
dos magistradas formaban parte del mismo. Por tanto, debemos considerar que existe una disponibilidad institucional para radicar la discriminación por razón de género en el acceso a los puestos de máxima responsabilidad de la judicatura y en los órganos colegiados de decisión.

\section{Feminización de la justicia desde la perspectiva de sus propios actores}

El Consejo General del Poder Judicial de España ha llevado a cabo diferentes consultas entre los componentes de la carrera judicial española, sobre temas de especial relevancia en cada momento, y que cuyos resultados se recoge en estudios e informes. Para esta investigación se han seleccionado las principales aportaciones que aparecen en ellos referidas a cuestiones de género.

Los documentos considerados han sido el VI Barómetro de Opinión de la Judicatura Española, ${ }^{7}$ la $V$ Encuesta a la Carrera Judicial y la Encuesta a la carrera judicial sobre conciliación de la vida familiar, ${ }^{8}$ así como de otros documentos que aparecen citados. Dicha información se completa con referencias a entrevistas realizadas a magistradas que han accedido a puestos de responsabilidad, y que han sido publicadas en la prensa española.

A continuación recogemos la opinión de los propios actores, jueces y magistrados de ambos géneros, sobre el proceso de feminización que está experimentando el sistema judicial español, respecto a las siguientes cuestiones:

7 El Barómetro tiene dos formatos distintos de preguntas: un primer tipo de pregunta a través de la cual el entrevistado tiene la opción de responder entre varias respuestas alternativas cerradas, o responder, con sus propias palabras, con preguntas abiertas. En un segundo tipo de pregunta se pide una evaluación expresada en forma de puntuación numérica por medio de una escala de cinco puntos, del 1 al 5 , y de once, del 0 al 10. Estos últimos resultados son presentados en forma de puntuación media. El método de recogida de información fue a través de una entrevista personal mediante cuestionario estructurado y precodificado (con algunas preguntas abiertas) administrado en el lugar de trabajo y con una duración media de 30 minutos. Al tratarse de un muestreo sobre población finita, y según los criterios del muestreo aleatorio simple, para un nivel de confianza del $95.5 \%$ (dos sigmas) y en la hipótesis más desfavorable $(\mathrm{p}=\mathrm{q}=50)$, el error de muestreo sería de $\pm 5 \%$. Las consultas anteriores fueron en los años 1984, 1987, 1990, 1993 y 1999. La última fue en 2003, y el trabajo de campo se realizó del 28 de abril al 20 de junio de 2003.

8 Dicha encuesta se ha llevado a cabo durante los meses de febrero y marzo del año 2008.

9 Hay que señalar que los miembros de la carrera judicial en España son reacios a hacer declaraciones públicas sobre el funcionamiento de dicha institución. Por eso hemos considerado unas fuentes de información valiosas las aparecidas en los medios de comunicación seleccionados. 


\section{1'. Aumento de la presencia de mujeres}

Hay que considerar la presencia de la mujer en la judicatura dentro de la normalización que supone el desarrollo de la sociedad democrática y dentro del reconocimiento efectivo y real de la igualdad de oportunidades y de una homogeneización en la distribución de roles, como la que se está produciendo en la sociedad española, aunque sea un a un ritmo más lento que el deseado.

Es una adaptación a la realidad social, una normalización, ya que en la sociedad estamos hombres y mujeres conviviendo, y en los órganos de enjuiciamiento es normal que estemos hombres y mujeres. Aporta además una serie de valores y de hábitos que están con más frecuencia en el acervo de la educación femenina: somos más concretas en las cosas, tenemos otra manera de dirigirnos a las personas, con más naturalidad, y eso facilita una mayor cercanía. Es indudable que me gustaría que hubiera más mujeres en la Audiencia, ya que la justicia sería más completa y se verían los problemas desde todos los prismas (Esther Erice, presidenta de la Audiencia Provincial de Navarra, Diario de Navarra, 22 noviembre de 2010).

Entre las razones explicativas del incremento de mujeres en la carrera judicial destacamos la opinión de los informantes de ambos sexos, acerca de la mejor predisposición de la mujer para preparar las pruebas selectivas. También existe una opinión compartida por jueces y magistrados de que la judicatura ofrece mayores posibilidades de promoción para la mujer, en comparación con otros cuerpos de la administración pública, lo que posiblemente se debe a que los requisitos de promoción son cada vez más objetivos. No obstante, esta opinión es sólo mantenida por el $43 \%$ de las mujeres y $42 \%$ de los hombres. Por el contrario, la afirmación referente a la facilidad de acceso a la carrera judicial, así como que exista una menor discriminación hacia la mujer por el hecho de serlo, no son compartidas, y las respuestas obtenidas reflejan disconformidad y un bajo nivel de aceptación (Cuadro 1).

\section{2a. Discriminación para poder acceder a los niveles superiores de la judicatura}

Al igual que sucede en la administración pública, a los puestos de mayor responsabilidad se accede después de muchos años de servicios y de experiencia probada, lo cual se consigue cuando se ha ido desempeñando puestos en diferentes jurisdicciones y destinos, o se tiene una larga trayectoria profesional reconocida, cuando se accede por el turno restringido, como catedrático de universidad en el área, despacho profesional, etc. y eso únicamente se ha conseguido a una edad avanzada. En 2014 había 614 juezas y magistradas con una edad comprendida entre los 51 y 60 años, y 73, entre 61 y 70 años, mu- 
chas de las cuales cumplen las condiciones para ocupar puestos de máxima responsabilidad.

Para Montserrat Comas — vocal del Consejo General del Poder Judicial, durante el periodo 2001-2008-: "Se trata de lograr una composición equilibrada, nunca hablamos de una paridad al $50 \%$ porque tampoco hay que ser drásticos. Ha faltado una política decidida. Simplemente con mirar la composición se puede hacer uno a la idea: no digo que los vocales estén en contra, pero romper las tendencias es mucho más complicado. Están acostumbrados a ver el mérito en los hombres" (El País, 22 abril 2008). La posibilidad de poder llegar a una paridad en los órganos superiores de la judicatura es un largo proceso. Las razones dadas por las juezas y magistradas son positivas y reflejan la esperanza de que finalmente se terminará consiguiendo.

No veo más cerrazón en la justicia que en otros ámbitos. En el Supremo, por ejemplo, creo que hay pocas mujeres por cuestión de edad. Además, sí que existe una inercia por la gran presencia de varones durante siglos. [...]. Hay un número importante de juezas en los órganos unipersonales que formarán parte más adelante de los órganos colegiados y porque desde el CGPJ sí que existe una intención y un trabajo de que los destinos dentro de la judicatura se atengan al currículum y la capacidad de cada uno (Esther Erice, presidenta de la Audiencia Provincial de Navarra, Diario de Navarra, 22 noviembre de 2010).

Se están haciendo esfuerzos para la igualdad, como la comisión al respecto del CGPJ - Consejo General del Poder Judicial—, que ofrece varios datos positivos. La paridad nos tiene que llevar un tiempo, o igual, viendo cómo vienen las futuras promociones, se da la vuelta a la proporción. Pero no se trata de lograr el 50\% entre hombres y mujeres, sino de dar más naturalidad a los puestos. Que no se valore el sexo, sino el bagaje personal (Mari Paz Benito, jueza decana de Pamplona, Diario de Navarra, 22 noviembre de 2010).

Esta opinión de no imponer una proporción igualitaria también se ha reflejado en la $V$ Encuesta a los miembros de la carrera judicial (Cuadro 2); la necesidad de una participación equilibrada por razón de género era valorada únicamente con 2,3 puntos sobre 10 , por un $89,7 \%$ de los participantes consultados. Esta concepción responde al carácter competitivo de las personas que forman este colectivo, que consideran que la promoción debe ser por méritos personales de preparación, capacidad y experiencia exclusivamente. 
3a. Dificultades para poder acceder a los niveles superiores de la judicatura, derivadas de la necesidad de conciliación familiar

La Encuesta sobre jornada laboraly conciliación familiar en el ámbito Judicial, ${ }^{10}$ elaborada por la Comisión Sindical de Jueces para la Democracia, señala que el 60\% de las mujeres tiene mayor dificultad para conciliar y que ello les afecta a su carrera profesional; mientras que en el caso de conciliar con la vida personal el impacto es similar en ambos géneros y afecta al $70 \%$ de los encuestados. Las dificultades para conciliar han perjudicado la vida profesional de un $39 \%$ de los encuestados, de los que el $58 \%$ son mujeres, sobre todo quienes son madres. En lo referente a puestos de responsabilidad, como señala Mercedes Guerrero, presidenta de la Audiencia Provincial de Álava: "En los puestos relevantes de la cúpula judicial las mujeres no estamos representadas [...], el problema es que nos resulta difícil conciliar la vida familiar y profesional. Las madres-magistradas tenemos que hacer malabares para atender a nuestros hijos y dedicarnos a esta profesión que tanto sacrificio exige" (El Correo.com, 2010).

La mayoría de las juezas y magistradas, pero especialmente las que tienen entre 30 y 40 años, o aquellas que tienen uno o dos hijos, según la Encuesta a la carrera judicial sobre conciliación de la vida familiar, consideran que el hecho de ser mujer condiciona negativamente el desarrollo de su carrera, pues tienen mayores dificultades por esta causa para competir y tener un desarrollo profesional en igualdad de oportunidades que sus compañeros. Esto lo refleja el que $39 \%$ de las consultadas afirman que ello les condiciona a la hora de solicitar una plaza con nombramiento discrecional o pedir un nuevo destino que suponga mayor dedicación.

Esta situación es similar a la que viven las mujeres profesionales de la administración de justicia en otros países (Bergallo, 2006; Gastiazoro, 2008). No obstante, dicha dificultad resulta algo menor a la hora de solicitar un destino que suponga un cambio de domicilio (33\%), lo cual indica que la mujer que accede a la carrera judicial tiene muy bien asumido que un ascenso en el escalafón, concretamente el paso de jueza a magistrada, implica en la mayoría de los casos necesariamente un cambio de localidad de residencia. Sin embargo, en los últimos años estamos observando nombramientos que implican

10 La encuesta ha sido remitida a todos los jueces y magistrados en servicio activo, y se ha realizado entre el 15 de marzo de 2012 y el 15 de abril de 2012, habiéndose recibido 346 respuestas de un total de 4.569 destinatarios, lo cual representa el 7,57\%. Los datos de los destinatarios son representativos del universo al que iba dirigida la encuesta, sin sesgos que desvirtúen los resultados obtenidos. 
cambio de residencia y son aceptados por las mujeres al igual como lo hacen sus compañeros.

No obstante, según datos recogidos en la misma Encuesta a la carrera judicial sobre conciliación de la vida familiar, el $71 \%$ de los encuestados (76\% mujeres y $64 \%$ hombres) considera que las cargas familiares deberían tenerse en cuenta a la hora de adjudicar un destino, y casi la totalidad (el 96,5\%) reconoce que el traslado por ascenso de categoría es un problema en su vida familiar. Por eso, el 98,3\% considera muy necesario incorporar medidas para mejorar la conciliación cuando se tengan hijos menores de tres años o el primer hijo, y en situaciones en las que un familiar está afectado por una enfermedad crónica o discapacidad, o en los casos en que el destino está lejos de la residencia familiar. Esta dificultad para conciliar la vida familiar y profesional vuelve a destacarse como un problema en la $V$ Encuesta a la Carrera Judicial, donde un $52 \%$ de los consultados considera que condiciona mucho y un $31 \%$ que algo.

\section{Conclusiones}

La feminización de la justicia española es ya una realidad, al igual que sucede en otros países. Este proceso se produce en toda la función pública, dado que la posibilidad de acceso a la misma depende principalmente de méritos personales, y es menor la discriminación por razón de género. Esta progresiva y constante incorporación de la mujer a la función pública en general y a la judicatura en particular debe explicarse desde la interrelación de factores en tres niveles, que son los que hacen factible el cambio (Saltzman, 1992):

- El micronivel, donde los estereotipos acerca de los roles que puede desempeñar la mujer, las creencias acerca de las diferentes habilidades y capacidades de hombres y mujeres, han evolucionado hacia la igualdad.

- El nivel medio: en este sentido, dicho estudio muestra cómo las diferentes instituciones y comunidades han realizado en las últimas décadas esfuerzos para promover la igualdad de oportunidades.

- Macronivel: los sistemas políticos y económicos, los sistemas de estratificación social o las ideologías que evolucionan más lentamente.

Las posibilidades de la mujer magistrada para acceder a los órganos superiores todavía está condicionada de alguna manera por la existencia entre algunos sectores de la magistratura que continúan prefiriendo varones para dichos puestos, y en otras ocasiones por los condicionantes que conlleva la necesidad de conciliar vida familiar y carrera profesional, lo cual constituye 
un impedimento para participar en concursos de promoción o tener que renunciar a puestos de designación discrecional. El techo de cristal continúa existiendo, no sólo por la resistencia institucional, en proceso de desaparición, sino porque la situación y el rol de mujer-madre les impide poder competir con sus compañeros, quienes están más libres de obligaciones familiares, aunque ellos en los cuestionarios afirmen que les condicionan igualmente.

Por tanto, el origen de esta discriminación profesional reside también en la desigualdad existente en el espacio doméstico, para el que la mujer ha sido educada y en donde la distribución de roles de antaño se sigue manteniendo (Bourdieu, 2005: 45; Touraine, 2007), de manera que a partir de que las nuevas generaciones se eduquen y asuman una distribución más igualitaria de funciones en la vida familiar, las oportunidades de la mujer en la vida laboral se incrementarán y se conseguirá una sociedad más justa.

La dicotomía doméstico-pública se refleja en las diferencias en el acceso a la administración en las escalas básicas, siendo un número creciente las mujeres, donde en las mismas promociones ellas han optado por puestos más estables, y los hombres por empleos con mayores remuneraciones y beneficios en la iniciativa privada. Sin embargo, esta tendencia podría revertirse debido a la crisis económica, dado que el alto desempleo en ambos sexos podría llevar a una mayor motivación de los hombres por puestos que anteriormente no resultaban de su interés así como por la búsqueda de una mayor estabilidad profesional.

\section{Bibliografía}

Amorós, Celia (2005), "Globalización y orden de género", en Amorós, Celia y Ana de Miguel [eds.], Teoría feminista: de la Ilustración a la globalización. De los debates sobre el género al multiculturalismo, vol. 3, Madrid: Minerva Ediciones.

Anagnostou, A. et al. (2004), Igualdad de oportunidades. Los discursos de las mujeres sobre avances, obstáculos y resistencias, Barcelona: Icaria.

Barberá, Ester (2005), Género y diversidad en un entorno de cambio, Valencia: Universidad Politécnica.

Beck-Gernsheim et al. (2001), Mujeres y transformaciones sociales, Barcelona: Hipatia.

Bergallo, Paola (2006), "¿Un techo de cristal en el poder judicial? La selección de los jueces federales y nacionales en Buenos Aires", en Luisa Cabal y Cristina Motta [comps.], Más allá del derecho. Justicia y género en América Latina, Bogotá, Colombia: El siglo del hombre.

Boigeol, Anne (2007), "So Many Women in the French Judiciary and so Few in the Upper Courts", Seminario Internacional sobre Mujeres en las Profesiones Jurídicas organizado por ELA (Equipo Latinoamericano de Género), Buenos Aires. 
Bourdieu, Pierre (2005), La dominación masculina, Barcelona: Anagrama.

Brunet, Ignasi et al. (2011), Sociología de las Organizaciones, Madrid: Universitas.

Bustillos, Julio (2011), El juez constitucional en el mundo. Perfil, carrera judicial, nombramiento, remuneración, desempeño y costos, México: Porrúa, IMDPC.

Carr-Rufino, Norma (1991), "US Women: breaking through the glass ceiling”, en Women in Management Review \& Abstracts, vol. 6, núm. 5, USA.

Castaño, Cecilia et al. (1999), Diferencia o discriminación, Madrid: Consejo Económico y Social.

Cavagna Martínez, Mariano et al. (1994), El poder judicial de la nación: una propuesta de reconversión, Buenos Aires: La Ley.

Cobo, Rosa (2010), “Globalización y nuevas servidumbres de las mujeres”, en De Miguel Álvarez, Ana y Celia Amorós Puente, Teoría feminista: de la Ilustración a la globalización (De los debates sobre el género al multiculturalismo.), vol. 3, Madrid: Minerva Ediciones.

Davison, Marilyn J. y Cary L. Cooper (1992), Shattering the glass ceiling, London: Paul Chapman.

Espuny Tomas, María Jesús et al. (2010), "Subiendo al estrado: mujeres y administración de justicia (1961-1966)”, en Rodríguez López, Rosalía y María José Bravo Bosch [eds.], Experiencias juridicas e identidades femeninas, Madrid: Dykinson.

Fix-Fierro, Héctor y Sergio López-Ayllon (2006), Del gobierno de los abogados al imperio de las leyes; estudios sociojurídicos sobre educación y profesión jurídicas en el México contemporáneo, México: UNAM.

Flores Sánchez, Aquiles (2012), “Mujeres en la judicatura federal en México (Preliminar para un diagnóstico sociológico de las féminas al servicio público de justicia y carrera judicial), en Revista del Instituto de la Justicia Federal, núm. 33, México.

Gastiazoro, María Eugenia (2008), "La concentración de las mujeres en la administración de justicia”, en Anuario del Centro de Investigaciones Jurídicas y Sociales (CIJS), núm. 11, Buenos Aires: Universidad Nacional de Córdoba.

Gastrón, Andrea (2009), "Género y argumentos de género en el Poder Judicial: Lo que muestran las sentencias judiciales en la Argentina”, en Revista Cientifica de UCES, vol. XIII, núm. 2, Buenos Aires: Universidad de Ciencias Empresariales y Sociales.

Holloway, Marguerite (1993), "A lab of her own", en Scientific American Magazine, noviembre, New York, USA.

Junqueira, Eliane (2003), "Women in the Judiciary: a Perspective from Brazil", en Shultz y Shaw, G. [eds.], Women in the World's Legal Professions: Oxford-Portland-Oregon: The Oñati International Institute for the Sociology of Law, Hart Publishing.

Kohen, Beatriz (2008), El género en la justicia de familia. Miradas y protagonistas, Buenos Aires: Ad Hoc.

Malleson, Jate (2007), "La justificación de la igualdad de género en la magistratura: por qué la diferencia no funciona”, en Revista Jurídica de la Universidad de Palermo, año 8, núm. 1 , Palermo.

Menkel-Meadow, Carrie (2002), “Portia in a Different Voice: Speculationson a Women's Lawyering Process”, en Ngaire Naffine [ed.], Gender and Justice, Washington: Ashgate. 
Convergencia Revista de Ciencias Sociales, núm. 70, 2016, Universidad Autónoma del Estado de México

Miller Moya, Luis Miguel (2004), "Participación laboral femenina y Estados de bienestar", en Revista Española de Investigaciones Sociológicas, núm. 108 (octubre-diciembre), Madrid.

Navarro-Guzmán, Capilla y Antonio Casero-Martínez (2012), “Análisis de las diferencias de género en la elección de estudios universitarios”, en Estudios sobre educación, vol. 22, Pamplona.

Osborne, Raquel (2005), "Desigualdad y relaciones de género en las organizaciones: diferencias numéricas, acción positiva y paridad", en Política y Sociedad, vol. 42, núm. 2, Madrid.

Perry, Bárbara (1991), A "Representative" Supreme Court? The Impact of Race, Religion, and Gender on Appointments, Nueva York: GreenwoodPress.

Robles García, María Pilar (2004), “Mujer y justicia”, en Humanismo y Trabajo Social, núm. 3, León, España: Universidad de León.

Rueda Romero, Paulino (2009), La administración de justicia en el Perú: problema de género, Lima: Universidad San Martín de Porres.

Saltzman, Janet (1992), Equidad y género. Una teoría integrada de estabilidad y cambio, Madrid: Cátedra.

Sánchez-Apellániz, Mercedes (1997), Mujeres, dirección y cultura organizacional, Madrid: CIS y Federación de Mujeres Directivas, Ejecutivas, Profesionales y Empresarias (FEDEPE).

Sarrio, Maite et al. (2002), "El techo de cristal en la promoción de las mujeres", en Revista de Psicología Social, vol. 17, núm. 2, Santiago de Compostela.

Telo, María (1966), "La evolución de los derechos de la mujer en España”, en A.A.V.V., La mujer española: de la tradición a la modernidad (1960-1980), Madrid: Tecnos.

Touraine, Alain (2007), El mundo de las mujeres, Barcelona: Paidós.

Weber, Max (1993), Economía y Sociedad, México: Fondo Cultura Económica.

Wirth, Linda (2002), Romper el techo de cristal. Las mujeres en puestos de dirección, núm. 58, Madrid: Ministerio de Trabajo y Asuntos Sociales de España, Colección Informes OIT.

\section{Recursos electrónicos}

Lynn, Martin (1991), “The three levels of the glass ceiling: Sorcerer's apprentice to through the looking glass”, vol. I, núm. 8, septiembre. Disponible en: www.cyberwerks.com/ dataline/mapping/thethree.html [16 de abril de 2008].

Ribas Monet, Antonieta (2014) "Desigualdades de género en el mercado laboral: un problema actual”. Disponible en: http://dea.uib.cat/digitalAssets/128/128260_4.pdf [24 de agosto de 2015].

\section{Otras fuentes de información}

Consejo General del Poder Judicial (2010), Acuerdo de 25 de febrero de 2010, del Pleno del Consejo General del Poder Judicial, por el que se aprueba el Reglamento 1/2010, que regula 
la provisión de plazas de nombramiento discrecional en los órganos judiciales (Boletín Oficial del Estado del 5 de marzo de 2010).

Consejo General del Poder Judicial (2013), Plan de Igualdad de la Carrera Judicial, Madrid: Comisión de Igualdad.

Consejo General del Poder Judicial (2003), Sexto Barómetro de Opinión de la Judicatura Española, Madrid: Demoscopia (dirección: José Juan Toharia).

Consejo General del Poder Judicial (2005), Informe sobre la estructura demográfica de la Carrera Judicial, a 1 de enero de 2005, Madrid: Servicio de Planificación y Análisis de la Actividad Judicial.

Consejo General del Poder Judicial (2014), Informe sobre la estructura demográfica de la Carrera Judicial, a 1 de enero de 2014, Madrid: Servicio de Planificación y Análisis de la Actividad Judicial.

Consejo General del Poder Judicial (2008), Encuesta a la carrera judicial sobre conciliación de la vida familiar, Madrid: Servicio de Planificación y Análisis de la Actividad Judicial, Cuarto Informe.

Consejo General del Poder Judicial (2010), V Encuesta a la Carrera Judicial.

Instituto Nacional de Estadística, Anuario Estadístico de España. Años 1961, 1977, 1982, 1992, 2002 y 2013. (Enseñanzas Universitarias.)

Jefatura del Estado, Fuero de los Españoles, Madrid: BOE 18 julio de 1945.

Jefatura del Estado, Ley de 18 de diciembre de 1950 por la que se regula la Escuela Judicial, Madrid: BOE del 19 de diciembre.

Jefatura del Estado, Ley 56/1961, del 22 de julio, sobre derechos politicos profesionales y de trabajo de la mujer, Madrid: BOE del 24 de julio de 1961.

Jefatura del Estado, Ley 96/1966, del 28 de diciembre, suprimiendo la limitación establecida en el apartado c) del número dos del artículo tercero de la Ley de 22 de julio de 161 sobre derechos de la mujer, Madrid: BOE del 29 de diciembre de 1966.

Jefatura del Estado, Ley Orgánica 3/2007, del 22 de marzo, para la Igualdad Efectiva de Hombres y Mujeres, Madrid: BOE del 23 de marzo de 2007.

Jueces para la Democracia (2012), La Encuesta sobre jornada laboral y conciliación familiar en el ámbito Judicial, Comisión sindical.

Ministerio de Trabajo y Asuntos Sociales (2007), Plan Estratégico de Igualdad de Oportunidades (2008-2011).

Ministerio de Política Territorial y Administración Pública. Resolución del 20 de mayo de 2011, de la Secretaría de Estado para la Función Pública, por la que se publica el Acuerdo del Consejo de Ministros de 28 de enero de 2011, por el que se aprueba el I Plan de Igualdad entre mujeres y hombres en la Administración General del Estado y en sus Organismos Públicos. (BOE de1 junio 2011).

Correo.com 9 de octubre de 2010

Diario de Navarra, 22 de noviembre de 2010

El País, 22 de abril de 2008 


\section{Tabla 1}

Forma de acceso a la carrera judicial por sexo

\begin{tabular}{lcccc}
\hline \multicolumn{1}{c}{ Forma de acceso } & Mujeres & Hombres & Mujeres & Hombres \\
\hline $\begin{array}{l}\text { Turno de juristas de reconocida } \\
\text { competencia 6 años }\end{array}$ & 63 & 142 & 60 & 132 \\
\hline $\begin{array}{l}\text { Turnos juristas contencioso-administrativo } \\
311.3 \text { y 6 }\end{array}$ & 5 & 13 & 5 & 12 \\
\hline $\begin{array}{l}\text { Turno de juristas } 10 \text { años civil art. 313.3 } \\
\text { y } 6\end{array}$ & 16 & 18 & 17 & 17 \\
\hline $\begin{array}{l}\text { Turno de juristas } 10 \text { años civil C-P- art. } \\
313.3 \text { y 6 }\end{array}$ & 26 & 29 & 23 & 26 \\
\hline Turno juristas 10 años penal art. 313.6 & 18 & 23 & 11 & 18 \\
\hline Turno juristas 10 años social art. 313.6 & 13 & 19 & 9 & 14 \\
\hline $\begin{array}{l}\text { Turno de juristas de reconocida } \\
\text { competencia 10 años }\end{array}$ & 49 & 136 & 47 & 131 \\
\hline $\begin{array}{l}\text { Turno de juristas de reconocida } \\
\text { competencia 15/20 años }\end{array}$ & & 11 & & 12 \\
\hline $\begin{array}{l}\text { Pruebas especialización contencioso- } \\
\text { administrativo }\end{array}$ & 3 & 9 & 2 & 9 \\
\hline Pruebas selectivas especialización social & 1 & 1 & & 1 \\
\hline Disposición transitoria 17 de la L.O.P.J. & 2 & 8 & 2 & 8 \\
\hline $\begin{array}{l}\text { Nombrado por el turno de Comunidades } \\
\text { Autónomas }\end{array}$ & 6 & 14 & 6 & 14 \\
\hline $\begin{array}{l}\text { Turno jurídico militar } \\
\text { Turno libre }\end{array}$ & 2212 & 1831 & 2247 & 1824 \\
\hline \begin{tabular}{l} 
Total \\
\hline
\end{tabular} & 2414 & 2259 & 2429 & 2223 \\
\hline
\end{tabular}

Fuente: Consejo General del Poder Judicial. Informe sobre la estructura demográfica de la Carrera Judicial, a 1 de enero de 2005 y 2014. 


\section{Tabla 2}

\section{Plantilla de jueces y magistrados por sexo y grupo de edad}

\begin{tabular}{lcccccc}
\hline & \multicolumn{3}{c}{1 enero 2005 } & \multicolumn{3}{c}{1 enero 2014 } \\
\hline & Mujeres & Hombres & Total & Mujeres & Hombres & Total \\
\hline 1857 & 2368 & 4225 & 2661 & 2558 & 5219 \\
\hline $\begin{array}{l}\text { Edad media } \\
\text { (años) }\end{array}$ & 39,62 & 46,73 & 43,6 & 44,72 & 50,49 & 47,55 \\
\hline $\begin{array}{l}\text { Antigüedad } \\
\text { (años) }\end{array}$ & 9,97 & 15,29 & 12,95 & 14,06 & 18,9 & 16,43 \\
\hline
\end{tabular}

Fuente: Consejo General del Poder Judicial. Informe sobre la estructura demográfica de la Carrera Judicial, 1 de enero de 2005 y 2014.

\section{Tabla 3}

\section{Proporción de mujeres jueces y magistrados por órgano de destino}

\begin{tabular}{ll}
\hline Tribunal Supremo & 11,7 \\
\hline Audiencia Nacional & 35,9 \\
\hline Juzgado Central de lo Contencioso Administrativo & 45.5 \\
\hline Tribunal Superior de Justicia & 33,6 \\
\hline Audiencia Provincial & 33,9 \\
\hline Juzgado de lo Penal & 59,0 \\
\hline Juzgado de los Contencioso Administrativo & 41,9 \\
\hline Juzgado de lo Social & 53,0 \\
\hline Juzgado de Vigilancia Penitenciaria & 59,2 \\
\hline Juzgado de Menores & 58,2 \\
\hline Juzgado de Primera Instancia e Instrucción & 65,1 \\
\hline Juzgado de Primera Instancia & 59,4 \\
\hline Juzgado de Instrucción & 55,4 \\
\hline Registro Civil Exclusivo Único & 60,0 \\
\hline Juzgado Decano Exclusivo & 16,7 \\
\hline Juzgado de lo Mercantil & 26,6 \\
\hline Juzgado de Violencia sobre la Mujer & 66,7 \\
\hline Tribunal Superior de Justicia pendiente de plaza & 64,9 \\
\hline
\end{tabular}

Fuente: Consejo General del Poder Judicial. Informe sobre la estructura de la Carrera Judicial, 1 de enero de 2014. 


\section{Cuadro 1}

\section{Razones explicativas del incremento de mujeres en la judicatura española}

\section{Cuestión planteada:}

¿Manifieste su conformidad/disconformidad con las

Mujeres

$(\%)$

siguientes posibles explicaciones del aumento de la

presencia femenina en la carrera judicial?

Las mujeres preparan las oposiciones con más intensidad que los hombres

La Judicatura ofrece mejores posibilidades de promoción a la mujer que en una empresa privada o en otros cuerpos de la administración

Existe menor discriminación contra la mujer en los tribunales de acceso a la judicatura que en otros tribunales de la administración

La Judicatura es una oposición más fácil de preparar que otras
53

55

Hombres

$(\%)$

$43 \quad 42$

Las cifras se refieren al \% de personas consultadas que han respondido afirmativamente al ítem con la respuesta "Mucho" o "Bastante".

Fuente: Sexto Barómetro de Opinión de la Judicatura Española, p. 30. 


\section{Cuadro 2}

Criterios selectivos propuestos por jueces y magistrados para la selección de los presidentes de los Tribunales Superiores de Justicia de las Comunidades Autónomas, Presidencias de las Salas, Presidencias de Audiencias Provinciales y Decanos de Jueces

\begin{tabular}{lcc}
\hline & $\begin{array}{c}\text { Valoración } \\
\text { de } 0 \text { a } 10\end{array}$ & $\begin{array}{c}\% \text { que } \\
\text { valora }\end{array}$ \\
\hline Antigüedad en el escalafón & 7,2 & 92,9 \\
\hline Antigüedad en el órgano a presidir & 6,4 & 91,5 \\
\hline Experiencia en el órgano colegiado & 7,0 & 91,8 \\
\hline Experiencia en el orden jurisdiccional correspondiente & 7,9 & 91,4 \\
\hline Plan de gestión del órgano a presidir de los candidatos & 5,5 & 88,7 \\
\hline $\begin{array}{l}\text { Entrevista en el CGPJ del candidato/a para exposición de } \\
\text { méritos/defensa de idoneidad }\end{array}$ & 3,3 & 91,4 \\
\hline Entrevista de presentación de méritos e idoneidad sea pública & 3,6 & 90,6 \\
\hline Participación equilibrada por razón de género & 2,3 & 89,7 \\
\hline
\end{tabular}

Fuente: V Encuesta a la carrera judicial 2010, p. 18.

Carlos Gómez Bahillo. Doctor en Ciencias Políticas y Sociología. Catedrático acreditado en Ciencias Sociales en la Universidad de Zaragoza. Líneas de investigación: sociología económica, políticas públicas, sociología de las organizaciones. Publicaciones recientes: Calidad de vida en las personas con inteligencia limite. Evaluación y propuesta de una Guia de indicadores para las organizaciones, Madrid: Universitas (2012); "La formación profesional en el sistema productivo", en A. Trinidad Requena y F. J. Gómez González, Sociedad, familia y educación. Una introducción a la Sociología de la Educación, Madrid: Tecnos (2012); "La orientación laboral ante la demanda de nuevos perfiles profesionales”, en A. Fabra Galofre y A. Giménez Adelantado [eds.], El cambiante mundo de las organizaciones. Teoria, metodología e investigación, Castellón de la Plana: Universidad Jaime I (2013).

Carmen Elboj Saso. Doctora en Sociología. Profesora titular de Sociología de la Educación y Catedrática acreditada en Ciencias Sociales (Universidad de Zaragoza). Líneas de investigación: socialización preventiva de violencia de género, minorías culturales, comunidades de aprendizaje. 
Publicaciones recientes: Pulido, C., Elboj, C. Campdepadrós, R., Cabré, J., Exclusionary and Transformative Dimensions: Communicative Analysis Enhancing Solidarity Among Women to Overcome Gender Violence. Qualitative Inquiry. Disponible en: http://qix.sagepub.com/content/ea rly/2014/06/17/1077800414537212 (2014); Gómez, A., Elboj, C. \& Capllonch, M., "Beyond Action Research. The Communicative Methodology of Research", en International Review of Qualitative Research, vol. 6, núm. 2 (2013); Elboj, C., Pulido, M. \& Welikala, T., "Las Tecnologías de la Información y la Comunicación en la salida del aislamiento rural. El caso de Ariño", en Scripta Nova, vol. XVII, núm. 427 (2013).

Celia Marcén Muñío. Doctora por la Universidad de Zaragoza. Programa de Sociología: ciudad, cultura y desarrollo. Profesora tiempo completo (Universidad San Jorge, Zaragoza). Líneas de investigación: sociología del deporte, género. Publicaciones recientes: Marcén, C., Gimeno, F. y Gómez, C., "Socioeconomic status, parental support, motivation and self-confidence in youth competitive sport", en Procedia-Social and Behavioral Sciencias, 82 (2013); Elsevier; Marcén, C., Gimeno, F. y Gómez, C., "Etnography as a linking method between Psychology and Sociology", en Procedia-Social and Behavioral Sciencias, 82 (2013); Elsevier; Marcén, C., Gimeno, F. y Gómez, C., "Evaluación del constructo 'apoyo parental' en jóvenes deportistas de competición”, en Prisma Social Revista de Investigación Social, 9, Fundación IS+D para la Investigación Social Avanzada (2012).

Recepción: 11 de octubre de 2014.

Aprobación: 31 de agosto de 2015. 